\title{
Guilt and Pro-Social Behavior amongst Workers in an Online Labor Market
}

\author{
Dr. Moran Blueshtein \\ Naveen Jindal School of Management \\ University of Texas at Dallas \\ USA
}

\begin{abstract}
Do workers in online labor markets feel guilt after behaving unethically? Recent laboratory studies with student participants have found that participants who made a financial gain from an unethical decision were more likely to subsequently engage in a prosocial behavior than participants who did not engage in that unethical decision. This inconsistency in preferences was attributed to guilt, whereby violations of a social norm induce a feeling of guilt that motivates a pro-social behavior. We find that these results do not hold in an online labor market setting, namely on Amazon Mechanical Turk (MTurk). Instead, MTurk workers who chose to be truthful despite the loss of potential gains were also the ones who were more likely to subsequently donate to a charity. Our study may have important implications to understand whether prior research on pro-social and unethical behavior extends to real-life business settings.
\end{abstract}

Keywords: Business Ethics, Experiment, Guilt, Online Labor Market, Prosocial Behavior

\section{Introduction}

Does guilt motivate prosocial behavior in online labor market settings? Emotions of guilt may arise from a violation of an important norm in a blameworthy manner (Ortony, Clore, \& Collins, 1988; Ketelaar\& Todd, 2001; Tangney \& Dearing, 2002), which may elicit feelings of tension and remorse (Lewis, 1987; Tangney, 1999).Evidence for moral consequences of guilt has been provided by a series of incentivized laboratory experiments, where participants were paid based on their decisions in the study. Ketelaar \& Au (2003) showed that people acted more pro-socially in social dilemma games after an autobiographical recall procedure inducing feelings of guilt or after making an unfair offer. Similarly, Nelissen, Dijker \& De Vries (2007) found that an induction of guilt increased cooperative behavior.

In a more recent paper, Gneezy, Imas, \& Madarasz (2014) — henceforth GIM — presented results of a classroom experiment where participants were first asked to choose whether to lie to increase their earnings, and then were given the option to donate to a charity. They find that those who chose unethically (to lie) were more likely to donate to a charity than those who made an ethical decision (were truthful). This suggests that norm violations induce a feeling of guilt due to which people exhibit an endogenous inconsistency in social preferences-and thus choose to behave prosaically following an earlier unethical decision. The objective of this paper is to test if these results hold also in an online labor market setting. To our knowledge, this is the first experiment to directly test for guilt-induced behaviors in an online labor market environment.

Online labor markets, such as Amazon's Mechanical Turk (MTurk), are becoming an increasingly popular means for conducting behavioral research. Online labor markets have several important advantages over laboratory or classroom experiments with student subjects. First, laboratory studies can be prohibitively expensive because they require participants to make substantial effort to participate in the study, and participants need to be compensated for the same. Second, online labor markets allow recruiting larger sample sizes and subjects that are much more diverse than student subjects in university laboratories. Third, the complete anonymity of online experiments may also reduce the risk of experimenter (demand) effects in which the subjects try to produce the effect they believe the experimenters expect. Last, and perhaps most important, the fact that subjects recruited from online labor markets are already in work environments may make the results of online studies more relevant for understanding behavior of individuals in a real life business environment. 
An increasing body of research testifies for the validity of data collected by Mturk. Rand (2012) surveys series of replication studies showing that data from Mturk is qualitatively consistent with data from offline laboratories. Similarly, Horton Rand \& Zeckhauser (2011) replicated some classic experiments in behavioral economics and found the results to be consistent with the lab results ${ }^{1}$. Crump et al (2013), on the other hand, replicated a diverse body of tasks from experimental psychology and found mixed results: while some of replications were qualitatively successful, others, however, have revealed disparity between laboratory results and online results.

We too find that the results from Mturk differ from those collected in an offline classroom setting. Contrary to GIM, we find consistency in preferences for individuals. In this online labor market environment, individuals who chose to lie and increase their gains at the expense of another individual were less likely to donate to charity than those who chose to be truthful and sacrifice a portion of their gains to benefit another individual. In addition, donation rates of individuals were similar regardless of whether or not they previously faced an ethical dilemma. Thus, our results suggest that among Mturk workers, there is no evidence that guilt motivates pro-social behavior.

\section{Experimental design}

Participants in the study were recruited on Mturk from the U.S worker population. To be eligible to participate in the study, all participants had to clear several attention check questions to demonstrate that they understood the instructions. A total of 240recruits were eligible to participate in this study. They were randomly assigned either to the Main Treatment (120 participants) or to the Reversed Treatment (120 participants). In each treatment group, half of the participants were randomly assigned a role of a Sender, and the other half a role of a Receiver. Each participant received 20 cents as a participation fee. In addition, participants were told that they could make an additional payment of up to 30 cents, depending on the decisions made in the study ${ }^{2}$. See Table 1 for an overview of the treatments

Table 1: Overview of the Treatments

\begin{tabular}{|l|l|l|}
\hline Treatment & Role & Number of participants \\
\hline \multirow{3}{*}{ Main Treatment } & Sender & 60 \\
\cline { 2 - 3 } & Receiver & 60 \\
\hline \multirow{2}{*}{ Reversed Treatment } & Sender & 60 \\
\cline { 2 - 3 } & Receiver & 60 \\
\hline
\end{tabular}

In the Main Treatment, we implemented a two-stage experiment design similar to GIM. First, Senders could choose to lie at the expense of the Receiver in order to increase their earnings (Deception Game). Next, Senders were given the option to donate to a charity. For the Deception Game we followed a game similar to Gneezy (2005). This deception game was modified in GIM to fit a classroom setting. We chose to use a framework similar to that of the original (Gneezy (2005) deception game as it was easier to implement in an online setting. In this two-player deception game, the Sender is told the outcome of the roll of a six-sided dice (in our experiment the outcome was 3). The Sender is then required to send a message to the Receiver and inform her about the outcome of the die roll. The Sender could choose between a true message ("The outcome from the roll of the 6sided die is 3") and a misleading message ("The outcome from the roll of the 6-sided die is 2").

The Sender in this setting has a monetary incentive to lie: If the Receiver chooses the true outcome of the die (3), then the Sender will earn 20 cents and the Receiver will earn 30 cents. However if the Receiver chooses any other number as the outcome of the die, then the Sender will earn 20 cents, and the Receiver will earn 30 cents. In other words -the Sender would earn 10 cents more and the Receiver 10 cents less than if the Receiver chose the correct number.

After the Senders sent the message to the Receivers, Senders were given the option to donate 2 cents to the charity "Doctors without Borders". 2 cents represent $20 \%$ of the potential gains from lying, which is the same donation ratio that was used in GIM. Senders were informed that "Doctors without Borders is an international humanitarian aid organization providing medical aid to children and women in war-torn regions".

\footnotetext{
${ }^{1}$ The authors showed that subjects (1) reverse decisions in response to how a decision-problem is framed, (2) have pro-social preferences and (3) respond to priming by altering their choices.

${ }^{2}$ These payment amounts follow the compensation recommended by the Guidelines for Academic Requesters (see http://wiki.wearedynamo.org/index.php/Guidelines_for_Academic_Requestersand also

http://wiki.wearedynamo.org/index.php?title=Fair_payment)
} 
It is important to note that, as in GIM, when Senders made their decisions in the Deception Game they did not know that they would later be given an option to donate a portion of their earnings in the first stage to a charity. After Senders and Receivers made their decisions, we implemented the choice of the Receivers. From the payments to the Senders who agreed to donate to the charity, we further subtracted that donation. Lastly, we made a donation to the charity at a sum equal to the total donation amounts. To further examine whether guilt motivates prosocial choices we also conducted a Reversed Treatment (not conducted in GIM). In the Reversed Treatment, the order of the stages of the Main Treatment was reversed. First, Senders were given the option to donate 2 cents to the charity, and then they played the Deception Game as described above. As before, the second stage of the game was not known to the players ahead of time - when Senders were given the option to donate 2 cents to the charity they did not know that they would later play a Deception Game.

\section{Experiment Results}

Figure 1: Fraction of Senders who Donated by Message Type - Current Study vs. GiM.

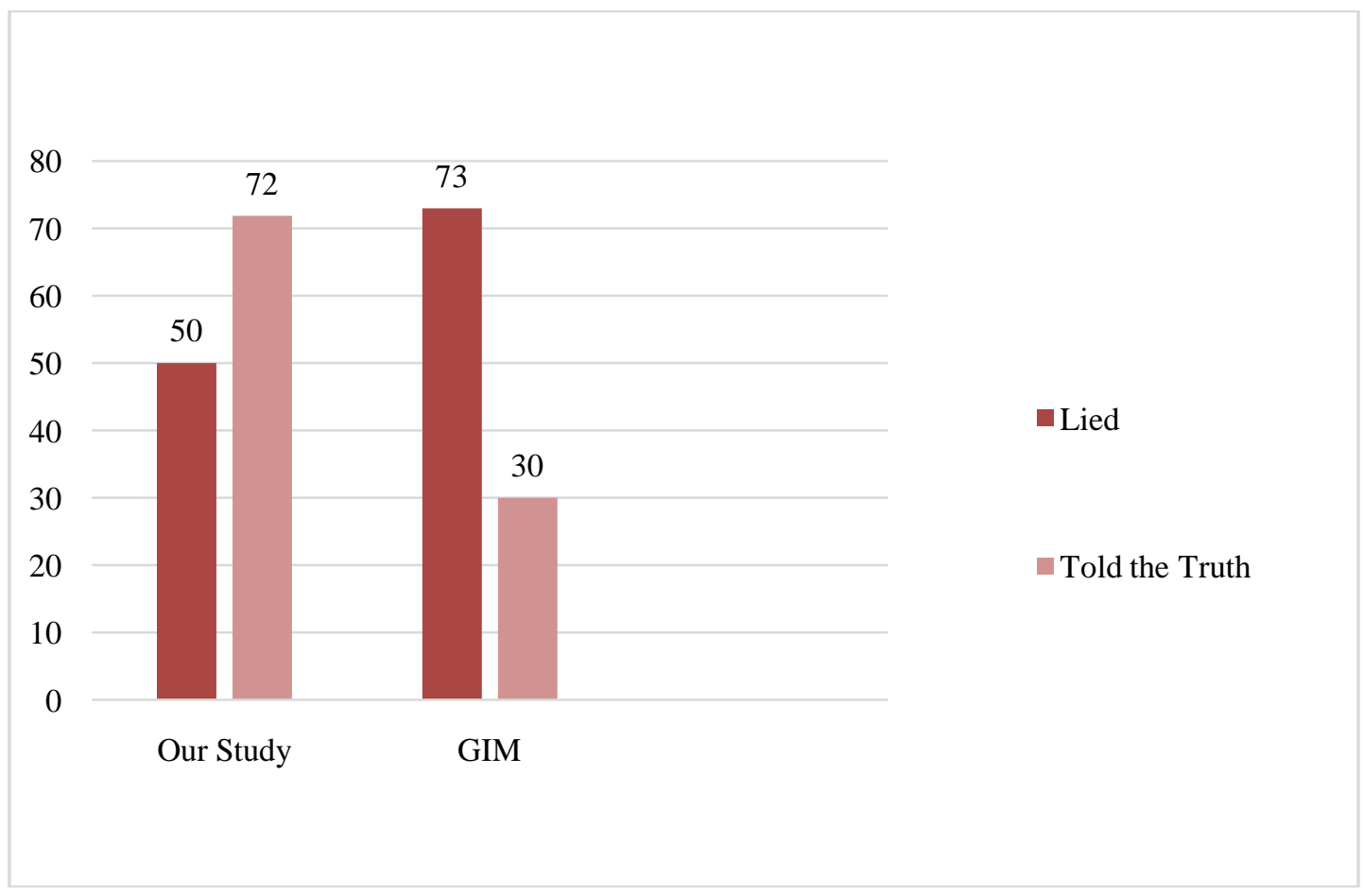

Figure 1 presents results for the fraction of Senders in the Main Treatment who donated depending on their decision in the Deception Game. We find that 50\% (14) of the Senders who chose to lie in the Deception Game, followed with a donation to the charity, compared with $72 \%$ (23) of the Senders who chose to be truthful. In fact, the difference is even weakly significant $(\mathrm{p}=0.08,2$-sided test). That is, participants in the Main Treatment who initially chose to lie in order to potentially earn 10 cents more from lying were subsequently less likely to donate to a charity compared with participants who chose to send a truthful message in the Deception Game. Figure 1 also presents the results for the fraction of Senders in GIM who donated depending on their decision in the Deception Game. As can be seen, in their study, 30\% of the participants who were truthful chose to donate, compared with $73 \%$ of those who lied ( $\mathrm{p}<.001,2$-sided test). Comparing our results with theirs, we see that the results in GIM do not hold in our setting. In particular, not only can we reject their hypothesis that initial unethical behavior leads to subsequent pro-social behavior, but in fact the consistency in ethical and pro-social behavior is marginally significant. Donation rates for the Main Treatment and Reversed Treatment are presented in Figure 2. 
Figure 2: Fraction of Senders who Donated by Treatment

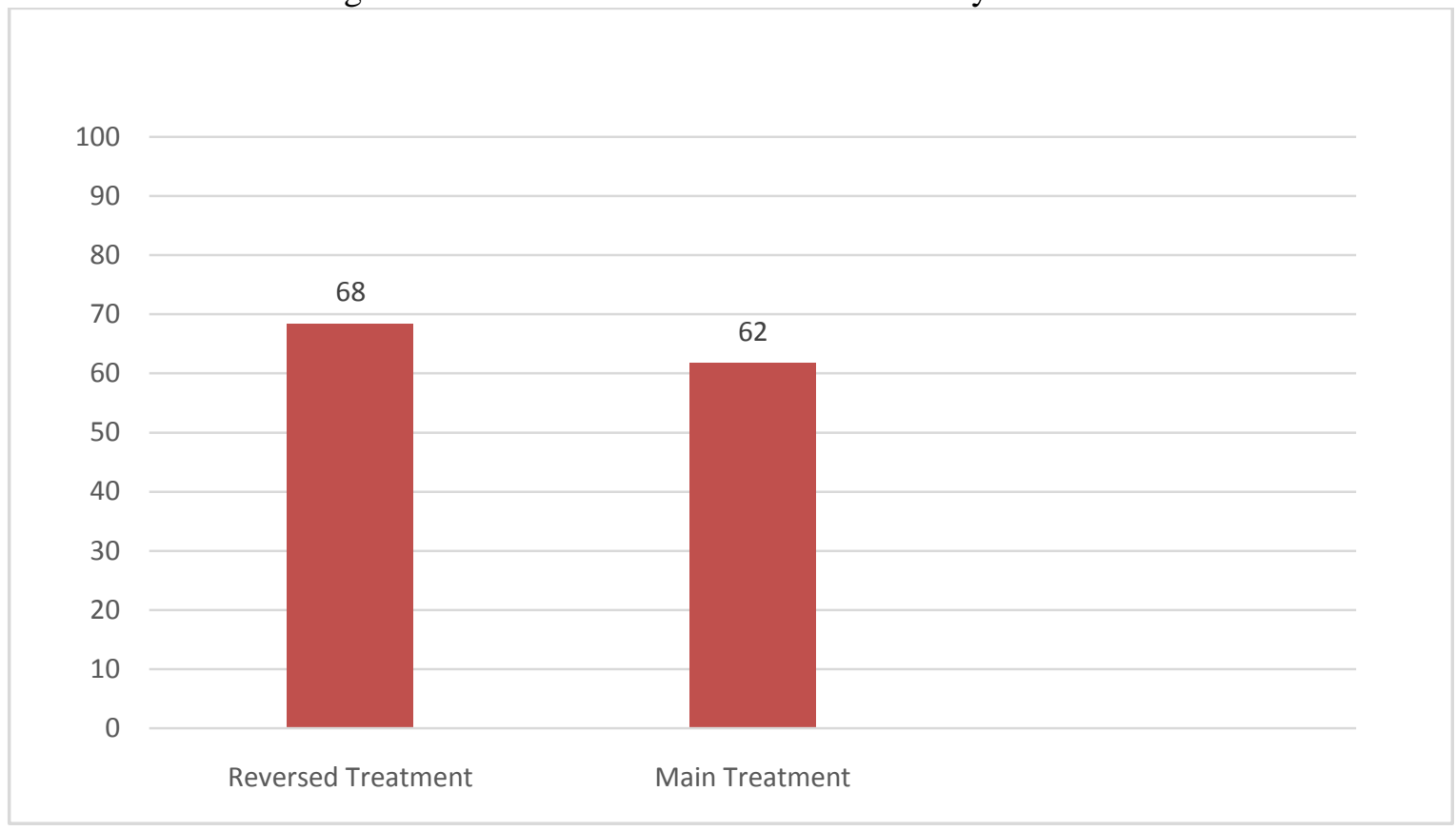

In the Main Treatment, 62\% (37) of the Senders chose to donate to the charity compared with 68\% (41) of the Senders in the Reversed Treatment. These differences are not statistically significant ( $\mathrm{p}=0.44,2$-sided test). That is, donation rates of participants who played a Deception Game before making a donation decision, were not statistically different from donation rates of participants who were first required to make a donation decision (without knowing that they would play a Deception Game later).Thus, we again reject the hypothesis posited in GIM.

Figure 3: Fraction of Senders who Lied by Donation Decision - Reversed Treatment

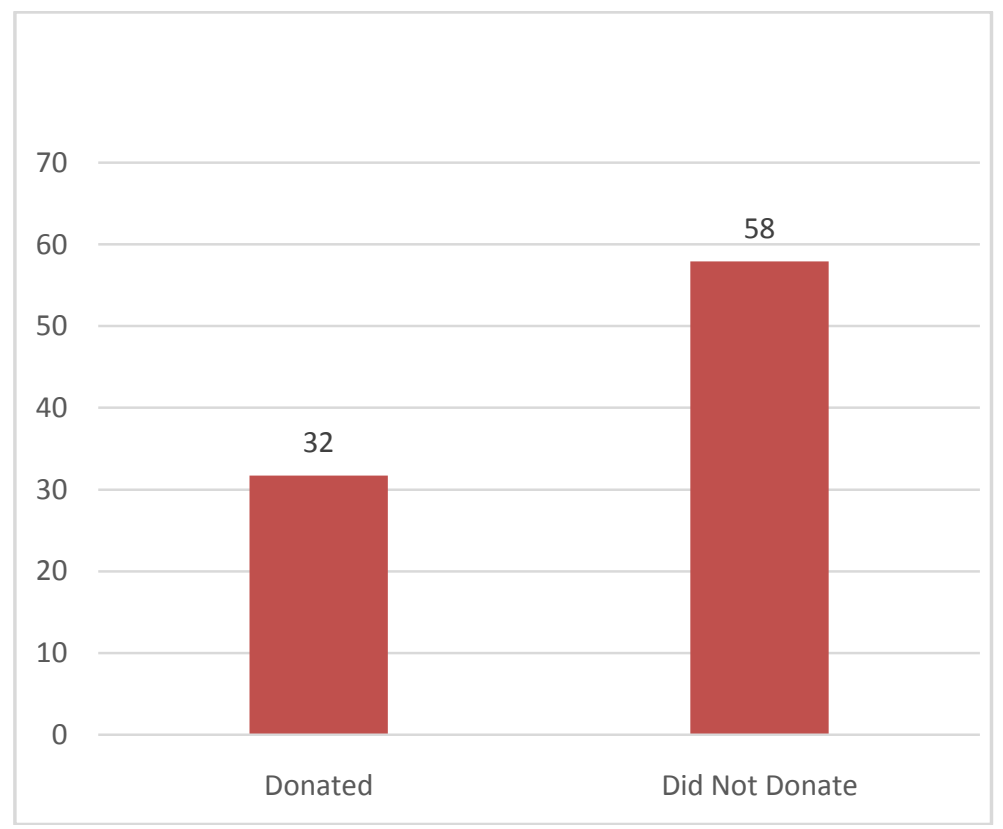

Figure 3 presents results for the fraction of Senders in the Reversed Treatment who lied depending on their earlier donation decision. We find that $32 \%$ (13) of the Senders who chose to donate to the charity subsequently chose to lie in the Deception Game, compared with $58 \%$ (11) of the Senders who chose not to donate. 
These differences are marginally significant ( $\mathrm{p}=0.054,2$-sided test). That is, lying rates of participants who donated earlier were lower than those of participants who chose not to donate. Thus, we again find consistency in ethical and pro-social behaviors.

\section{Discussion and Conclusions}

Recent behavioral literature suggests that people will behave pro-socially after making an unethical choice because of guilt. Our experiment casts doubt as to whether these results hold outside classroom and laboratory experiments with student participants. In this paper, we adopted an experimental framework similar to GIM, who have previously shown that guilt after an unethical choice leads to pro-social behavior in a classroom study. However, we obtained very different results in an online labor market environment.

We find that participants who lied also subsequently donated less, while those who were truthful, subsequently donated more. Unlike the GIM classroom experiment, our results are consistent with a theory of heterogeneity in pro-social preferences. According to this theory, individuals are classified into "types" - some types are more ethical or prosocial than others. Therefore, their prosocial behavior is consistent with their preferences across decisions. Individuals who behave morally (do not lie), on average, also donate more to a charity than individuals who choose to lie.

To further verify the robustness of our results we conducted a Reversed Treatment, where Senders were given the option to donate before they knew they would later face an ethical dilemma. Our results support our previous findings of preference consistency. First, we find that donation rates were not statistically different between the Main Treatment and the Reversed treatment. That is, that facing an ethical decision before making a donation decision had no effect on donation decisions. This suggests that it is the type of the individual and not her previous decision that influenced her decision whether or not to donate. Second, we find that lying rates of participants who donated earlier were lower than those of participants who chose not to donate. This suggests that preference consistency holds independent of the sequence of the decisions.

Overall, our results suggest that among Mturk workers there is no evidence that guilt motivates pro-social behavior. It is possible that unlike in a classroom environment, when subjects make decision in their work environment, they view the task from an economic frame of mind (Horton Rand \& Zeckhauser (2011)) and therefore their decisions are less influenced by emotions like guilt. These findings can be relevant to understanding ethical and pro-social behavior in business settings. As such, guilt may not play a significant role in real world business settings and, thus, may be unlikely to lead to subsequent pro-social behaviors. Rather, the intrinsic ethical nature of a person also determines their tendency for pro-sociality.

\section{References}

Crump, M.J.C., McDonnell, J.V. \&Gureckis, T.M. (2013) Evaluating Amazon's Mechanical Turk as a Tool for Experimental Behavioral Research. PLoSONE 8(3): e57410. https://doi.org/10.1371/journal.pone.0057410

Gneezy, U.(2005). Deception: The Role of Consequences. American Economic Review 95 (1): 384-94.

Gneezy, U., Imas, A., \&Madarász K (2014) Conscience accounting: Emotion dynamics and social behavior. Management Science. 60(11): 2645-2658

Horton, J.J., Rand, D.G. \&Zeckhauser, R.J. (2011) Experimental Economics 14(3): 399-425.

Ketelaar, T., \& Au, W. T. (2003). The effects of guilt on the behavior of uncooperative individuals in repeated social bargaining games: An affect-as-information interpretation of the role of emotion in social interaction. Cognition \& Emotion, 17, 429 -453 .

Ketelaar, T., \& Todd, P. M. (2001). Framing our thoughts: Ecological rationality as evolutionary psychology's answer to the frame problem. In H.R Holcomb, III(ED.), Conceptual challenges in evolutionary psycology. Dordrecht: Kluwer

Lewis, H. B. (1987). Shame and the narcissistic personality. In D. L. NatJimon (Ed.), The many faces of shame (pp. 93-124). New York, NY: Guilford Press.

Nelissen, R. M. A., Dijker, A. J., \& De Vries, N. K. (2007). How to turn a hawk into a dove and vice versa: Interactions between emotions and goals in a give-some dilemma game. Journal of Experimental Social Psychology, 43, 280 -286.

Ortony, A., Clore, G. L., \& Collins, A. (1988). The cognitive structure of emotions. New York, NY: Cambridge University Press.

Rand, D. J. (2012). The promise of Mechanical Turk: How online labor markets can help theorists run behavioral experiments. Journal of Theoretical Biology 299: 172-179.

Tangney, J. P. (1999). The self-conscious emotions: Shame, guilt, embarrassment, and pride. In T. Dalgleish\& M. Power (Eds.), Handbook of cognition and emotion (pp. 541-568). Chichester, England: Wiley.

Tangney, J. P., \& Dearing, R. L. (2002). Shame and guilt. New York, NY: Guilford Press. 